\title{
Counterfeit medicines: a pilot study for chemical profiling employing a different proposal of a usual technique
}

\author{
Maíra Kerpel dos Santos*a,d, Alessandro Kahmann ${ }^{\mathrm{b}}$, Luiza Manica Caffarate ${ }^{\mathrm{a}}$, Laura Ribas Ucha ${ }^{\mathrm{a}}$, Renata P. Limberger ${ }^{\mathrm{a}, \mathrm{d}}$, \\ Rafael S. Ortiz ${ }^{\mathrm{c}, \mathrm{d}}$ \\ ${ }^{a}$ Toxicology Analysis and Research Laboratory, Federal University of Rio Grande do Sul, Department of Pharmacy, \\ Porto Alegre, 90610-000, RS, Brazil; ${ }^{b}$ Institute of Mathematics, Statistics and Physics, Federal University of Rio Grande \\ (FURG), 96214020, Rio Grande, Brazil; ' ${ }^{B}$ Brazilian Federal Police, Regional Superintendence of Rio Grande do Sul, \\ 90160-093, Porto Alegre, RS, Brazil; ' National Institute of Forensic Science and Technology (INCT Forensics), Porto \\ Alegre, RS, Brazil.
}

\section{*Corresponding author: mai.krps@gmail.com}

Gas chromatography (GC) is a gold standard technique used in forensic laboratories, including for the characterization of counterfeit medicines. When coupled simultaneously to flame ionization (FID) and mass detector (MS) allow the identification and quantification of medicines and drugs employing a single method, besides permitting the application of chemometric tools for forensic intelligence purposes. Here is presented a pilot study that developed and applied a simple qualitative method for the analysis of irregular medicines using a simple extraction procedure followed by GC-FID/MS analysis, with application of chemometric tools. The main purpose was to identify similarities between the all compounds detected in the irregular medicines allowing the traceability of illicit producers with the creation of a common data base. Seized medicines obtained from different producers were extracted with methanol and submitted to ultrasound and centrifugation. $1 \mathrm{~mL}$ was then injected into the GC through simultaneous detection by FID and MS. Through the analyses it was verified that different producers of counterfeit medicines labeled as Sibutramine, added a mixture of Caffeine and Benzocaine to its formulation, respecting the same ratio of 2.2:1. Hierarchical Cluster Analysis (HCA) was able to confirm these results, showing the presence of both falsifications in the same cluster, representing the best tool to identify similar characteristics among the samples. Other interesting finding was the use of Fluoxetine as a falsification of counterfeit medicines labeled as Sibutramine and Diethylpropion. Another seized sample labeled as "Nobésio Forte", marketed as a mix of stimulants, showed mainly Caffeine and Lidocaine in its formulation. The pilot project applied primarily to 45 samples of counterfeit medicines containing amphetamine-type stimulants and antidepressants, showed the capability of perform the chemical profiling of counterfeit medicines in the solid form - powder, capsules and tablets. Further analysis can be performed for different types of medicines in solid form using the developed method, allowing the construction of a single database to perform the chemical profiling of counterfeit medicines, helping forensic intelligence to track the illicit producers.

Keywords: irregular medicines, chemical profile, GC-MS, GC-FID, hierarchical cluster analysis. https://doi.org/10.22456/2527-2616.107986

\section{Introduction}

Investigations and analyses of counterfeit medicines have been rising in recent years following the growing of medicines falsification worldwide [1,2]. Chemical profiling of counterfeit medicines plays an important role in forensic intelligence in order to identify the different types of falsification and illicit producers [3]. Several methods have been employed for chemical analysis of counterfeit medicines, being most common the chromatographic and spectroscopic [2]. The use of multivariate statistical analysis combined with results obtained by analytical techniques is a valuable trend for chemical profiling. These combinations bring relevant information of medicines and drugs, by focusing in similarities and dissimilarities of active ingredients, excipients, residual solvents and etc. [4,5]. Similarities in the chemical constitution can be shown using exploratory tools such as Principal Component Analysis (PCA) and Hierarchical Cluster Analysis (HCA), indicating the same producer for samples from different apprehensions, or even different medicines, helping forensic intelligence [6]. Analysis of excipients likewise can raise important information about the counterfeit medicines production [5], highlighting correlations between different samples, which can use the same formula or mixture of components for the production of distinct medicines, as was identified for counterfeit medicines of Cialis and Viagra, reported by Ortiz et al, 2013

Here was proposed a pilot project through gas chromatography with simultaneous analysis by flame ionization and mass spectrometer detector, followed by application of chemometric tools of HCA and PCA, to determine similarities between irregular medicines seized by Brazilian Federal Police of Rio Grande do Sul. The main purpose was to develop and implement a simple method of GC to identify a standard mixture of substances used to produce different counterfeit medicines in solid form, permitting its traceability. 


\section{Material and Methods}

\section{Seized medicines}

45 solid samples of counterfeit medicines (tablets and capsules) from different batches were seized in the South of Brazil, in 2017, by Brazilian Federal Police (Porto Alegre, Rio Grande do Sul). The counterfeit medicines comprised mainly amphetamine-type-stimulants, such as Sibutramine, Fenproporex, Ritalin, Mazindol and Amfepramone; antidepressants (Fluoxetine), and some non-identified formulas like "Nobésio Forte" (Table 1).

Table 1. Irregular medicines seized by Brazilian Federal Police of Rio Grande do Sul.

\begin{tabular}{cccl}
\hline Medicines & API & Manufacturer & $\begin{array}{c}\text { Solid oral } \\
\text { dosage form }\end{array}$ \\
\hline Fenproporex & Fenproporex & Unidentified & Capsule \\
Sibugras & Sibutramine & Unidentified & Capsule \\
Sibutramina & Sibutramine & Unidentified & Capsule \\
Nobésio & Unidentified & Unidentified & Tablet \\
Forte & & & \\
Dimagrir & Mazindol & Gadol ${ }^{\circledR}$ & Capsule \\
Reductil & Sibutramine & Phidia & Capsule \\
& & Pharma ${ }^{\circledR}$ & \\
Ritaline & Methylphenidate & Novartis® & Tablet \\
Dualid & Diethylpropion & Aché ${ }^{\circledR}$ & Capsule \\
Biomag & Sibutramine & Aché ${ }^{\circledR}$ & Capsule \\
Fluoxetina & Fluoxetine & Biosintética ${ }^{\circledR}$ & Capsule \\
Fluoxetina & Fluoxetine & Genom ${ }^{\circledR}$ & Capsule \\
\hline
\end{tabular}

* API: Active Pharmaceutical Ingredient.

\section{Sample preparation}

First, tablets and capsules were individually weighted, in triplicate. Then, the tablets had their shell removed (if present) and the capsules were opened, had their content removed, followed by homogenization. The mixture powder of each medicine was weighted considering $10 \%$ of its total weight, in triplicate. The powder was then transferred to a polypropylene tube in which $5 \mathrm{~mL}$ of Methanol was added. The tubes were submitted to a vortex for $1 \mathrm{~min}$, added to ultrasound for $10 \mathrm{~min}$ and centrifuged at 3000 r.p.m. for 5 minutes. $1 \mathrm{~mL}$ of supernatant was added to a $2 \mathrm{~mL}$ vial and transferred to the chromatograph for automatic injection. The volume of injection was $1 \mu \mathrm{L}$.

\section{Gas Chromatography (CG)}

The analyses were conducted using a GC-MS/FID (7890B/5977B) Agilent Technologies (CA, USA) equipped a Combipal CTC Analytics (Basel, Switzerland), and a two-way splitter to connect the GC column to mass spectrometer (MS) and flame ionization (FID) detectors. For chromatographic separation was employed a DB5-MS column $(30 \mathrm{~m}$ x $0,25 \mathrm{~mm}$ x
$0,25 \mu \mathrm{m})$. Were also used connecting capillaries without a stationary phase, with dimensions of $0.10 \mathrm{~mm}$ I.D., 1 $\mathrm{m}$ long to MS; and $0.15 \mathrm{~mm}$ and $1.62 \mathrm{~m}$ long to FID. The final proportion MS:FID was 29:71. Mass detector was operated using $70-\mathrm{eV}$ (EI) with a source temperature, quadrupole and interface of $230^{\circ} \mathrm{C}, 150{ }^{\circ} \mathrm{C}$ and $280{ }^{\circ} \mathrm{C}$ respectively. The analyses were performed in Scan mode ranging $40-550 \mathrm{~m} / \mathrm{z}$. The injector was set at $280{ }^{\circ} \mathrm{C}$ using a split of 1:20. Oven temperature was programmed to starting at $100{ }^{\circ} \mathrm{C}$ to $280{ }^{\circ} \mathrm{C}$ with an increase of $20^{\circ} \mathrm{C} / \mathrm{min}$, and held for $2 \mathrm{~min}$; then starting at $280{ }^{\circ} \mathrm{C}$ to $310{ }^{\circ} \mathrm{C}$ with at an increase of $5{ }^{\circ} \mathrm{C} / \mathrm{min}$, and held for $17 \mathrm{~min}$. The total run was $39 \mathrm{~min}$. Ultrapure helium was the carrier gas at $1 \mathrm{~mL} / \mathrm{min}$ for DB-5MS; 1.5 $\mathrm{mL} / \mathrm{min}$ for MS connecting capillary and $3.858 \mathrm{~mL} / \mathrm{min}$ for FID connecting capillary. FID detector temperature was set at $300{ }^{\circ} \mathrm{C}$. Synthetic air and Hydrogen at 300 and $30 \mathrm{~mL} / \mathrm{min}$ respectively, were used to keep the flame; and Nitrogen at $25 \mathrm{~mL} / \mathrm{min}$ was employed as a make-up gas.

\section{Chemometrics}

Determination of similarities between seized medicines and assessment of additional information from chemical analysis was employed by using Hierarchical Cluster Analysis (HCA) PASW Statistics ${ }^{\circledR}$, version $18 \circledR$ and Principal Component Analysis (RStudio, Version 1.2.5033, package stats).

\section{Results and Discussion}

The seized medicines analyzed comprised amphetaminetype stimulants from different producers such as Pharma Arte $^{\circledR}$, Gadol $^{\circledR}$, Aché $^{\circledR}$, Novartis ${ }^{\circledR}$, Phidia Pharma ${ }^{\circledR}$ and some unidentified companies, along with antidepressants from Biosintética ${ }^{\circledR}$ and Genom ${ }^{\circledR}$ (Figure 1). FID data was used for multivariate statistical analysis considering the peak areas of all the compounds identified in the chromatographic run from each medicine, and their respective retention times. In order to standardize which peaks would be considered in the chromatogram, a cutoff was applied to the peaks that presented a minimum of $0.02 \%$ of the largest peak area in the chromatogram. Simultaneously, the MS results were processed in order to identify the compounds, using a full scan analysis $(40-550 \mathrm{~m} / \mathrm{z})$ and considering the comparison with NIST library, literature data and the deconvolution of selected peaks.

By HCA it is possible to identify similarities between the seized samples through its proximity in the experimental space [8]. The proximity between the samples leads the formation of distinct clusters (groups), which are interpreted by analyzing a Dendrogram. After HCA analysis using FID data it was possible to identify the formation of seven clusters, as can be seen in Figure 2. Cluster $\mathrm{G}$ had the sample of Ritalin ${ }^{\circledR}$ which did not show the correspondent API Methylphenidate. However, Ritalin showed peaks of excipients degradation at 8.40, 9.00, 9.90 and $13.01 \mathrm{~min}$. 


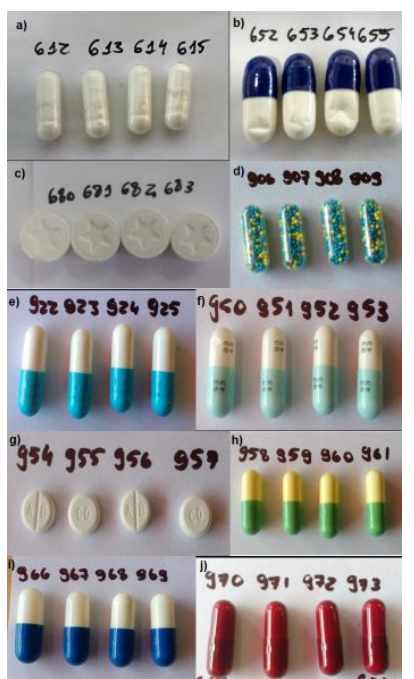

Figure 1. Pictures of medicines seized by Brazilian Federal Police of Rio Grande do Sul. a) Fenproporex; b) Sibutramine; c) Nobésio Forte; d) Dimagrir; e) Reductil; f) Fluoxetine (Biosintética); g) Ritalin; h) Fluoxetine (Genom); i) Biomag and j) Dualid.

Those retention times were not encountered in any of the other medicines, justifying the superior distance from the other groups. Cluster F, E and D were also formed for one type of medicine each, containing Dimagrir ${ }^{\circledR}$, Dualid $^{\circledR}$ and Nobésio Forte ${ }^{\circledR}$, respectively. Dimagrir ${ }^{\circledR}$ showed the correspondent API Mazindol at $17.7 \mathrm{~min}$. Dualid $^{\circledR}$ revealed the presence of Fluoxetine instead of Diethylpropion at a RT of 13.63 min. Nobésio Forte ${ }^{\circledR}$, a mixed formula without manufacturer identification, showed as main compounds Caffeine at $13.65 \mathrm{~min}$ and Lidocaine at $13.95 \mathrm{~min}$, respectively.

The most interesting result was encountered in Cluster C with samples of Sibugras ${ }^{\circledR}$ and Sibutramine ${ }^{\circledR}$, seized medicines from unidentified producers that presented the combination of Benzocaine at 11.898 -11.901 min and Caffeine at 13.65-13.652 min, instead of presenting Sibutramine as the API. It was also verified the proportion of addition of these compounds in both seized medicines, calculating the ratio between the two compounds. The results showed the same proportion of 2.2:1 (Benzocaine:Caffeine) in Sibugras ${ }^{\circledR}$ and Sibutramine ${ }^{\circledR}$. This preliminary result could indicate the ability of the method of grouping the samples using the applied criteria, beyond the focus on the API, identifying a mixture of substances that could be employing by the producers. Cluster $\mathrm{C}$ also showed the presence of Fluoxetine from Genom ${ }^{\circledR}$ and Biosintética ${ }^{\circledR}$. These samples remained at cluster $\mathrm{C}$ due to their similarity in the retention times (RT), since Fluoxetine showed a RT of $13.63 \mathrm{~min}$ closed to caffeine at $13.65 \mathrm{~min}$. The sample Biomag ${ }^{\circledR}$ was also present in Cluster $\mathrm{C}$ and however, can be easily seen that a lower Dendrogram cutoff at 4.9 (red line) could separate it in another cluster, not applied here due to the Parsimonious principle [6]. This sample showed a degradation product at $15.7 \mathrm{~min}$, without any correspondence with the API Sibutramine. Cluster B, close related to Cluster $\mathrm{C}$ was formed by the sample
Reductil $^{\circledR}$ and did not grouped with Cluster B due to the presence of a degradation product at 13.97 min derived from excipient degradation, beyond the peak at 13.63 min, attributed to Fluoxetine, instead of the correspondent API Sibutramine. Cluster A was formed by one compound at $12.2 \mathrm{~min}$ correspondent to the API Fenproporex.

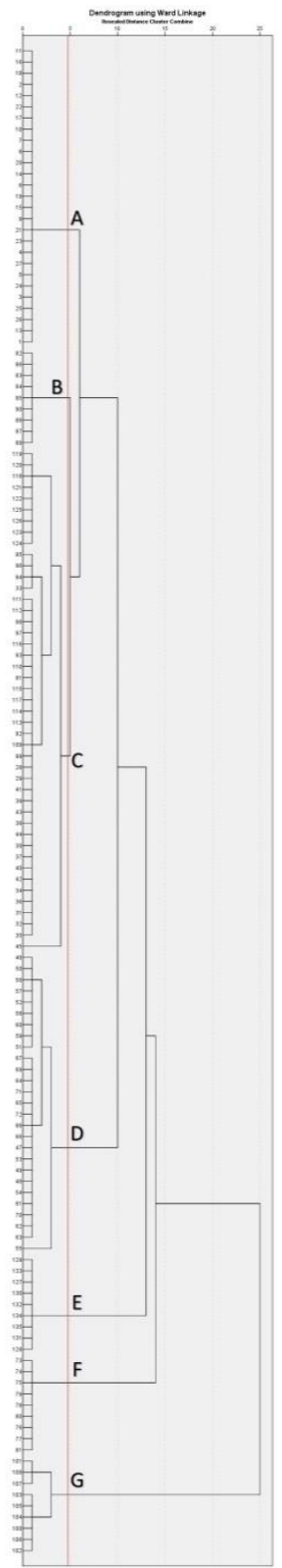

Figure 2. Dendrogram of 45 counterfeit medicines analyzed by GC-MS/FID in triplicate.

PCA showed the formation of four groups (Figure 3). Group I which consisted only of Ritalin ${ }^{\circledR}$. Group II which presented Dualid ${ }^{\circledR}$ and Group III which contained Dimagrir $^{\circledR}$, showing similar results to those obtained by HCA for the 3 samples, which also remained in separated clusters in HCA. However, group IV was composed by the rest of the seized samples with few non-zero values, that means, few compounds identified by the RT variable. Therefore, samples with non-zero 
value only in a restricted number of variables does not present significant distinction among other samples, explaining the formation of group IV. So, despite also showing the presence of the samples Sibugras ${ }^{\circledR}$ and Sibutramine ${ }^{\circledR}$ in the same group, the results encountered by PCA analysis did not showed significant information. Thus, considering the data base constructed with FID results and the main purpose of identify similar characteristics obtained from all the substances detected in the chromatogram, HCA showed better results when compared to PCA, even when applied to a small number of samples. Both medicines, Sibutramine ${ }^{\circledR}$ and Sibugras ${ }^{\circledR}$, showed peak areas in the same proportion and RT, indicating a high probability of production of both seized medicines using an equivalent formula, as was already detected in other studies with counterfeit medicines of Cialis and Viagra [7]. This information can be useful for forensic intelligence in order to track the illicit producers of these counterfeit medicines and can be further applied to other types of seized medicines that present a solid dosage form, contributing for the formation of a common database for police intelligence.

The samples analyzed showed degradation products, the API and mixtures of substances employed as a common formula for different medicines. Different from the usual methods, which search specifically for the API of each medicine to group the samples, the aim here was to obtain all the compounds present in the chromatograms and use these values to construct a database, targeting chemometric analysis. So, the purpose was not to compare the medicines against their authentic samples and find their API, but use the same extraction process and GC method for different solid materials, allowing the identification of similar formulas that could indicate a common origin between the seized medicines.

After the analysis, it was also noted that Fluoxetine, a Serotonin-Selective Reuptake Inhibitor used as antidepressant [9], it's being employed to falsify seized samples labeled as Sibutramine and Diethylpropion. Considering the results of this study and comparing with other studies performed by our research group [10], the target of falsification for the amphetamine-type stimulants now seems to be Sibutramine instead of Fenproporex. Since 2011, Sibutramine it's the only anorectic stimulant commercialized in Brazil according to National Health Surveillance Agency (ANVISA). [11]. Concerning the samples of "Nobésio Forte", which presented an indicative use for increase alertness in informal Brazilian market, especially for truck drivers $[10,12]$, the analyses showed the presence of Caffeine and Lidocaine, instead of any type of amphetamine-type stimulants such as, fenproporex, sibutramine or diethylpropion. Both anesthetics are commonly employed as adulterants in the production of cocaine, mainly to enhance and simulate its effects $[13,14]$.

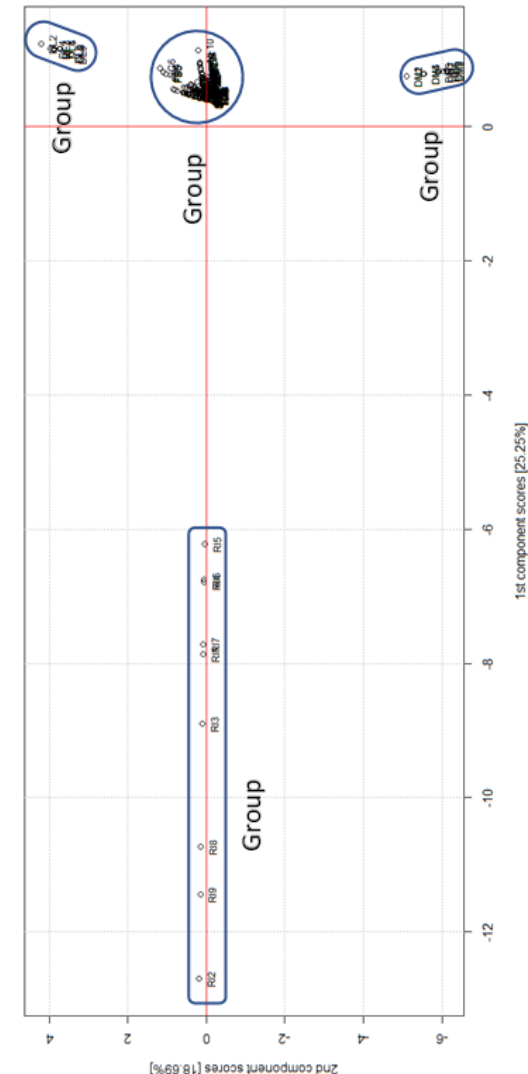

Figure 3. PCA analysis of the 45 counterfeit medicines analyzed by GC-FID/MS in triplicate.

\section{Conclusions}

The method using a simple extraction procedure followed by the analysis through GC and the simultaneous detection by FID and MS, was able to perform the chemical profiling of the counterfeit medicines analyzed. Thus, it can be further applied in the routine of forensic laboratories to analyze distinct seized solid materials - medicines and drugs - enabling the construction of a single database. Thus, allows the correlation of data from all seized samples by employing HCA as a complementary approach to the traditional applications of GC. In this case, the conditions for using the developed method is to detect all the constituents present in the chromatogram of seized solid samples following the criteria established - beyond the focus on the API, drafting a different perspective for chemical profiling using GC.

\section{Acknowledgments}

The authors are thankful to Brazilian Federal Police (Porto Alegre, Rio Grande do Sul) for technical support and to CAPES, CNPQ and INCT Forensic for financial support MCTI/CNPQ/CAPES/FAPSno16/2014 - INCT, CNPq 465450/2014-8.

\section{Conflict of interest}

The authors declare no conflicts of interest. 


\section{References}

1.WHO GSMS. WHO Global Surveillance and Monitoring System for substandard and falsified medical products [Internet]. 2017. Available from: http://www.who.int/medicines/regulation/ssffc/publica tions/GSMS_Report.pdf?ua=1.

2.Rebiere H, Guinot P, Chauvey D, et al. Fighting falsified medicines: The analytical approach. J Pharm Biomed Anal. 2017;142:286-306.

3.Dégardin K, Roggo Y, Margot P. Forensic intelligence for medicine anti-counterfeiting. Forensic Sci Int. 2015;248:15-32.

4.Custers D, Canfyn M, Courselle P, et al. Headspacegas chromatographic fingerprints to discriminate and classify counterfeit medicines. Talanta [Internet]. 2014;123:78-88. Available from: http://dx.doi.org/10.1016/j.talanta.2014.01.020.

5.Coelho Neto J, Lisboa FLC. ATR-FTIR characterization of generic brand-named and counterfeit sildenafil- and tadalafil-based tablets found on the Brazilian market. Sci Justice [Internet]. 2017;57:283-295. Available from: http://dx.doi.org/10.1016/j.scijus.2017.04.009.

6.Kahmann A, Anzanello MJ, Fogliatto FS, et al. Interval importance index to select relevant ATRFTIR wavenumber Intervals for falsified drug classification. J Pharm Biomed Anal [Internet]. 2018;158:494-503. Available from: https://doi.org/10.1016/j.jpba.2018.06.046.

7.Ortiz RS, Mariotti K de C, Fank B, et al. Counterfeit Cialis and Viagra fingerprinting by ATR-FTIR spectroscopy with chemometry: Can the same pharmaceutical powder mixture be used to falsify two medicines? Forensic Sci Int. 2013;226:282-289.

8.Ortiz RS, Mariotti K de C, Holzschuh MH, et al. Profiling counterfeit Cialis, Viagra and analogs by UPLC-MS. Forensic Sci Int. 2013;229:13-20.

9.Zavvari F, Nahavandi A, Goudarzi M. Fluoxetine attenuates stress-induced depressive-like behavior through modulation of hippocampal GAP43 and neurogenesis in male rats. $\mathbf{J}$ Chem Neuroanat [Internet]. 2020;103:101711. Available from: https://doi.org/10.1016/j.jchemneu.2019.101711.

10.Mariotti K de C, Ortiz RS, Souza DZ, et al. Trends in counterfeits amphetamine-type stimulants after its prohibition in Brazil. Forensic Sci Int. 2013;229:2326.

11.BRASIL. Resolução RDC número 52 de 6 de Outubro de 2011. Diário Of. 2011;53:1-3.
12.Zancanaro I, Limberger RP, Bohel PO, et al. Prescription and illicit psychoactive drugs in oral fluid-LC-MS/MS method development and analysis of samples from Brazilian drivers. Forensic Sci Int. 2012;223.

13.Conceição VN, Souza LM, Merlo BB, et al. Estudo do teste de Scott via técnicas espectroscópicas: Um método alternativo para diferenciar cloridrato de cocaína e seus adulterantes. Quim Nova. 2014;37:1538-1544.

14.De Carvalho TC, Tosato F, Souza, Lindamara M. et al. Thin layer chromatography coupled to paper spray ionization mass spectrometry for cocaine and its adulterants analysis. Forensic Sci Int. 2016;262:5665. 